\title{
Designing Search and Rescue Robots towards Realistic User Requirements
}

\author{
DOROFTEI Daniela ${ }^{1, a^{*}}$, MATOS Anibal ${ }^{2, b}$ and DE CUBBER Geert ${ }^{1, c}$ \\ ${ }^{1}$ Royal Military Academy, Department of Mechanics, Unmanned Vehicle Centre, \\ Avenue de la Renaissance 30, 1000 Brussels, Belgium \\ ${ }^{2}$ University of Porto, Faculty of Engineering, Ocean Systems Group, \\ Rua Dr. Roberto Frias 4200-465 Porto, Portugal \\ a *daniela.doroftei@rma.ac.be, banibal@fe.up.pt, ${ }^{c}$ geert.de.cubber@rma.ac.be
}

Keywords: Robot mechanical design, user / system requirements engineering, search and rescue

\begin{abstract}
In the event of a large crisis (think about typhoon Haiyan or the Tohoku earthquake and tsunami in Japan), a primordial task of the rescue services is the search for human survivors on the incident site. This is a complex and dangerous task, which often leads to loss of lives among the human crisis managers themselves. The introduction of unmanned search and rescue devices can offer a valuable tool to save human lives and to speed up the search and rescue process. In this context, the EU-FP7-ICARUS project [1] concentrates on the development of unmanned search and rescue technologies for detecting, locating and rescuing humans. The complex nature and difficult operating conditions of search and rescue operations pose heavy constraints on the mechanical design of the unmanned platforms. In this paper, we discuss the different user requirements which have an impact of the design of the mechanical systems (air, ground and marine robots). We show how these user requirements are obtained, how they are validated, how they lead to design specifications for operational prototypes which are tested in realistic operational conditions and we show how the final mechanical design specifications are derived from these different steps. An important aspect of all these design steps which is emphasized in this paper is to always keep the end-users in the loop in order to come to realistic requirements and specifications, ensuring the practical deployability [2] of the developed platforms.
\end{abstract}

\section{Introduction}

The ICARUS project [1] aims to bridge the gap between the research community and end-users, by developing a toolbox of integrated components for unmanned search and rescue (SAR). The objective of the ICARUS project is to develop robots which have the primary task of gathering data. The unmanned SAR devices are foreseen to be the first explorers of the area, as well as in situ supporters to act as safeguards to human personnel. In order not to increase the cognitive load of the human crisis managers, the unmanned SAR devices will be designed to navigate individually or cooperatively and to follow high-level instructions from the base station [3]. The robots connect wirelessly to the base station and to each other, using a wireless self-organising cognitive network of mobile communication nodes which adapts to the terrain. The unmanned SAR devices are equipped with sensors that detect the presence of humans and will also be equipped with a wide array of other types of sensors. At the base station, the data is processed and combined with geographical information, thus enhancing the situational awareness of the personnel leading the operation with in-situ processed data that can improve decision-making. The Haitian experience has shown the importance acquired by the geographic component in the management of human and technical resources in crisis situations. Similarly, it has highlighted that a suitable distribution (through interoperable standards) and real-time generation of thematic maps (demolished buildings, destroyed bridges, etc.) allows optimisation and interoperability of these resources and accelerates the access to victims. All this information will be integrated in existing C4I systems [3], used by the forces involved in the operations. This paper describes the user requirements for the different 
ICARUS developments [4]. The aim is to gather information from the end-users on the desired / expected capabilities of the ICARUS system.

\section{User identification \& requirements gathering}

A thorough understanding of the end-user community is a key preliminary factor in order to be able to define a correct set of end user requirements. In the case of ICARUS, it is clear that the tools to be developed are targeted towards the international SAR community. An important distinction to be made depends on the terrain where the rescue operations are taking place. There exists a separate urban SAR (USAR) and maritime SAR (MSAR) community, operating in a different environment.

The international USAR community is organized at UN-level via the INSARAG secretariat. INSARAG has established a world-wide network of USAR teams, developed a standardized set of Guidelines and established an External Classification (IEC) system for USAR teams [5]. INSARAG provides a single point of entry to access nearly the whole international USAR community.

For MSAR operations, ICAO and IMO are the international entities that coordinate all SAR global efforts of their member states. The SAR system, like any other system, has individual components that must work together to provide the overall service. Operationally, the global MSAR system relies upon States to establish their national MSAR systems and then integrate provision of their services with other States for world-wide coverage.

As the USAR and MSAR communities are quite separate, we recognized that it was required to foresee separate user contact and user requirement gathering approaches specifically targeted towards each of these distinct communities. An iterative information gathering approach was followed, where multiple draft documents were reviewed and validated by an end-user board, consisting of members of both the USAR and MSAR communities. The main information sources for these draft documents were data collected from previous user requirements documents, personal interviews with key stakeholders and online questionnaires targeted specifically at both communities. An important point in this approach was also the validation of draft user requirements through the presentation and discussion at key events where end-users are present, such as the RPAS2013 conference [6], the Sea Forum [7] and the INSARAG [5] Team Leaders Meeting, covering as such the air, marine and ground robotics users community.

\section{Main user requirements conclusions}

A complete overview of the user requirements for all ICARUS goes beyond the scope of this paper. In order to maximize the impact of the paper towards the scientific community, we focus on these requirements which are often overlooked by scientists developing robotic systems.

Fast deployment. Unmanned SAR tools which need to be deployed in a remote area, must meet the requirements of air transportability. This poses great constraints on the weight and size of all unmanned components. Goods to be transported over the air must fit in the cargo bay of standard aircraft used for rescue operations. Aircraft cargo space is very expensive, certainly at the moment of a crisis operation when many airplanes are demanded in a short period of time. As such, also the size of the package must be kept to a minimum. Realistically, it can be put that the whole rescue package should fit on 2 standard euro-pallets, which limits the dimensions to $120 \mathrm{~cm} \mathrm{x} 160 \mathrm{~cm} \times$ $95 \mathrm{~cm}$. The package must not only contain the robotic tools themselves, but also the tools to repair them. Moreover, the package may not contain any dangerous goods, to avoid problems and delays with customs. It must also be possible to deliver this package at the national airport, within 6 hours after getting notice of deployment. The weight of the total package is an important issue and must of course be brought to a minimum. The maximum mass for a package can be estimated at $100 \mathrm{~kg}$. This is the maximum weight for a package to still be offloaded from a cargo plane by 2 humans. If the mass exceeds this number, then a forklift is necessary, which is often problematic in crisis areas.

Manpower requirements. SAR teams are invariably faced with a massive overload of work, so "sacrificing" people to operate the robotic tools is not an easy compromise. The users were asked 
to indicate how many extra team members they would be willing to include in their teams for operating the unmanned platforms. As a general conclusion one it can be noted that no more than 2 people should be required to operate all robotic tools.

Energy requirements. Continuous electrical power supply is not a certainty in disaster areas. In general, SAR teams need to count on their own power sources. In general a power generator is used for these purposes, and -more and more - also solar panels. Care must be taken that the robotic tools do not require more electrical power (e.g. for recharging) than can be given by these power generators. The user survey showed that most teams have access to power generators of up to $2 \mathrm{kVA}$, so this should be seen as an upper limit for electrical power draw.

Water and dust resistance. SAR teams are often working in wet and dusty conditions. As a result, also the unmanned systems should be water and dust-resistant. To assess the required level of water and dust resistance, the end users were asked to indicate the desired level of water resistance they would desire for the different platforms, according to the Ingress Protection Rating or IP code. As a result of this study, the target IP level for outdoor aerial platforms was set at IP53, whereas ground platforms are rated at IP65 and marine platforms were rated at IP85.

Daytime and Nighttime operation. The end-users indicate that both ground and aerial systems should be able to operate in total darkness. This requirement is mostly relevant for the indoor platforms, as - in many cases - USAR operations are paused during the nighttime for security reasons, although some teams note that they work 24/7. Some USAR teams also report that the night time would be the ideal time for robot interventions, as it is calmer and unmanned vehicles could be less constrained by security problems. For MSAR applications, the possibility of nighttime operation is one of the most relevant features and selling points. Indeed, current (manned) operations almost always need to be halted overnight, but unmanned systems could go on through the night and thereby drastically improve the chances of survival of victims still in the water.

Autonomy requirements. The level of autonomy to be incorporated in the systems is a delicate exercise. Many end-users indicated us that in practical SAR operations, the robots will always need to be tele-operated for safety and legal reasons. Evidently, this request clashes with the desire of scientists wanting to equip their platforms with intelligent autonomous navigation systems. One must also not forget that legal issues also play a role in this matter. Allowing e.g. unmanned aircraft in airspace is already a delicate issue and allowing autonomous aircraft will be even more so. As a result, it must be foreseen that - at all time - the ICARUS aerial platforms can switch to complete tele-operation and act as Remotely Piloted Aircraft (RPA) in order not to limit their deployability.

Sensing requirements. The end-users were asked to indicate and prioritize the required sensing modalities they would like to see installed on the different systems. The results show that end-users value the visual contact with the victims (video cameras) and that the geo-referencing of the victims is also of high importance. The next more selected answers were related to detection sensors (infrared and other human detection sensors). Furthermore, structural 3D mapping for increasing the situational awareness and the presence of a microphone on ground platforms for communicating with trapped victims are requested by end-users.

Communication requirements. One must be aware that in a disaster-affected area, the local communication infrastructure is often largely dysfunctional. Often, mail and telephone connections do not work. Skype chat is one of the most robust services to keep a conversation. This clearly shows that ad-hoc communication tools are required.

Command and control requirements. Up until today, there are few hi-tech tools in a SAR context. This is mainly due to the fact that SAR workers are reluctant to introduce new technologies in the field, as the crisis environment is extremely technology unfriendly. Crisis managers are under huge stress to carry out a lot of work. As a result, all technology they are required to use must be made extremely user-friendly. This requirement calls for simple interfacing technologies, where most of the background processing tasks are hidden from the user, such that the user only has to give high-level (task) commands. 


\section{System and architecture requirements}

Gathering information from the user requirements and the development teams, system requirements and an architecture definition were obtained. The deployment scheme of the ICARUS architecture can be depicted by the scheme of Fig. 1 .

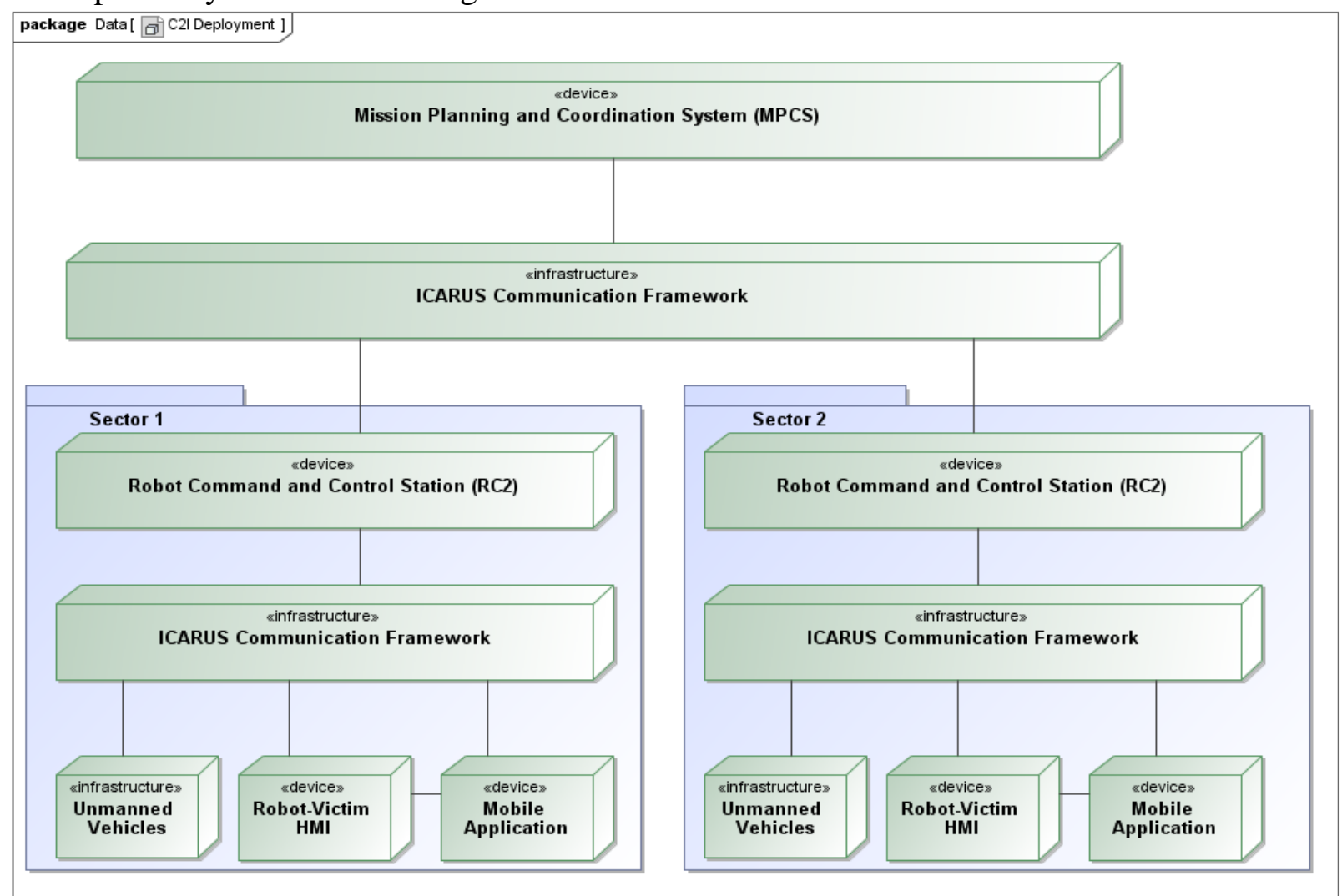

Fig. 1: ICARUS Deployment Architecture

The ICARUS Mission Planning and Coordination System (MPCS) [3] gets always deployed at the Crisis Coordination Center and performs the mission planning and coordination. Depending on the plan, an ICARUS team can perform mission-level activities commanded directly from the Crisis Coordination Center. In the case of an urban SAR operation, this will be the On-Site Operations and Command Centre, where also the Local Emergency Management Authority and crisis data providers will input their data and mission objectives. In the event of a maritime SAR operation, this central coordination system would be the Maritime Rescue and Command Centre.

In case of a crisis situation covering a wider area, the crisis coordination centre will in general divide the crisis area in sectors and assign incoming SAR teams to the different sectors based upon team capabilities and specific sector needs. The number of sectors can vary wildly based on the extent of the crisis. For reasons of clarity Fig. 1 sketches a situation with only 2 sectors, but the architecture can be extended easily. In this architecture, each sector receives its own Robot Command and Control Station (RC2) [3], which has to connect with the MPCS via the ICARUS communication Framework. Inevitably, this communication link will have to deal with constraints on the amount of data which can be sent over the communication link. The SAR Robot Operator uses the Robot Command and Control Station to control the (multiple) ICARUS unmanned vehicles through the ICARUS communication framework. Some ICARUS vehicles are equipped with a robot-victim HMI system, enabling disaster victims to send feedback (voice, video) from the victim back to the RC2. The Local Emergency Management Authority and the Crisis Data Providers and Crisis Stakeholders interact with the MPCS to input data, enabling the SAR mission planner to assign tasks and missions to the different ICARUS tools via the MPCS. In the field, SAR Field Teams and First responders are assisted by the ICARUS robots to rescue victims. They have mobile 
devices at their disposal, running mobile applications allowing them to read robot sensor data via the Communication Network and to contact the RC2 for requesting a change in robot tasking.

Following this architecture and the user requirements, system requirements are derived for each of the ICARUS platforms. Fig 2. gives a brief overview of each of these systems, referring the reader to reference documents to gain more information about each of the subsystems.

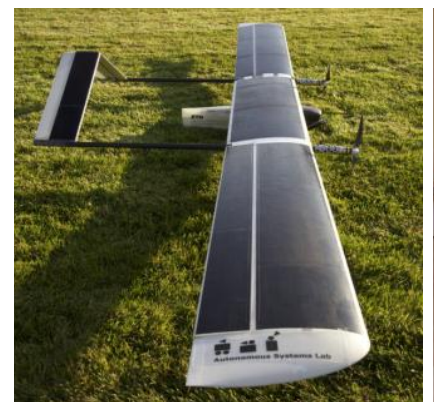

a)

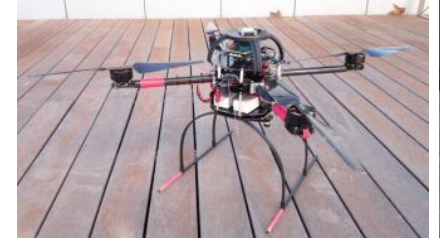

e)

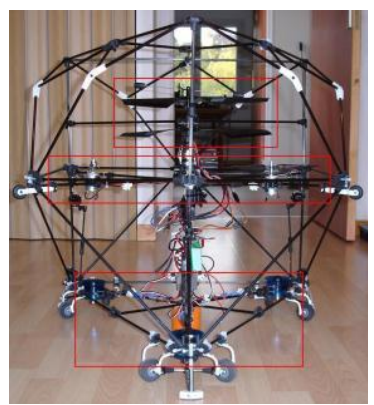

b)

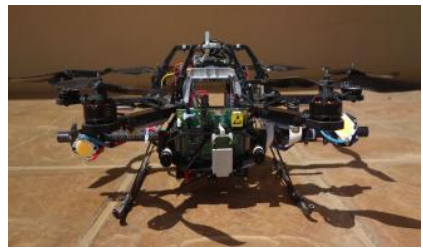

f)

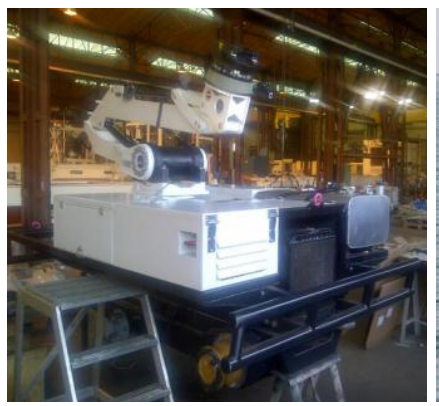

c)

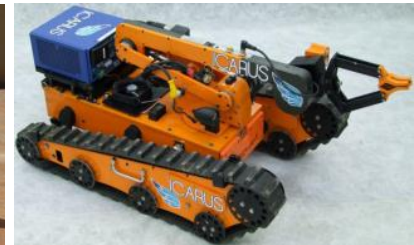

g)

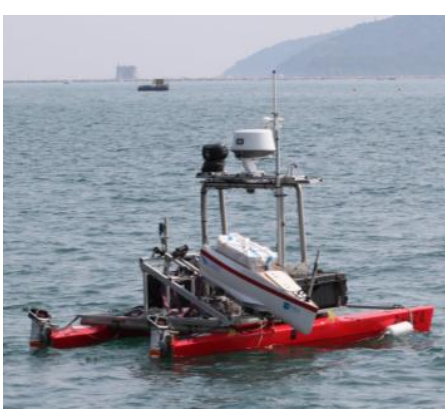

d)

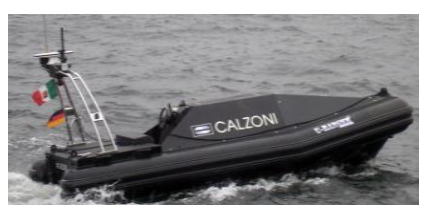

h)

Fig. 2: ICARUS Platforms: a) ETH Solar aeroplane [8], b) JmdTheque Gyropendulum [6],

c) META Large Ground Vehicle [9], d) INESC ROAZ II with Unmanned Capsule [10], e) ASCAMM quadrotor [6], f) Skybotix indoor rotorcraft [11], g) Allen Vanguard Digital Vanguard [9], h) Calzoni U-Ranger [12].

\section{Operational validation of the user requirements and conclusions}

It is often hard to express requirements without evaluating the practical operational repercussions of these requirements by doing field tests with the produced material. Therefore, the ICARUS consortium has opted to organize, together with the end users, operational trials already very early in the project stage, showcasing the capabilities of early developments and prototypes, in order to get valuable feedback from the end-users, allowing the designers to improve their systems and in order to allow the end-users to re-iterate their requirements. Fig. 3 shows some images of the land and sea trials and the ICARUS subsystems put to the test.

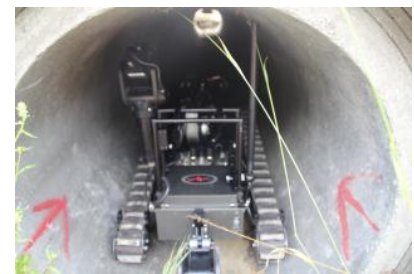

a)

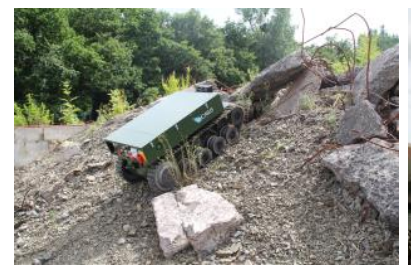

e)

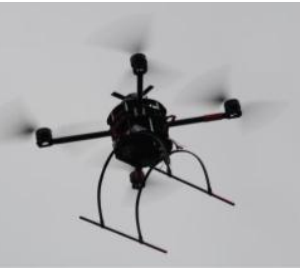

b)

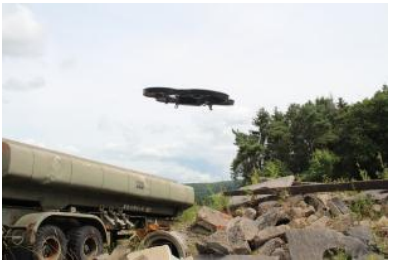

f)

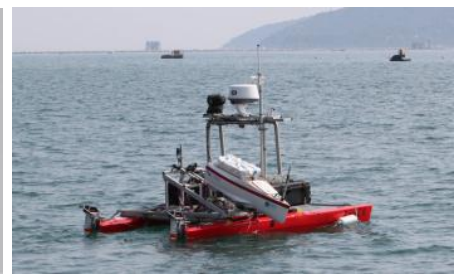

c)

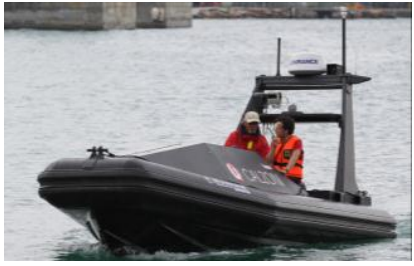

g)

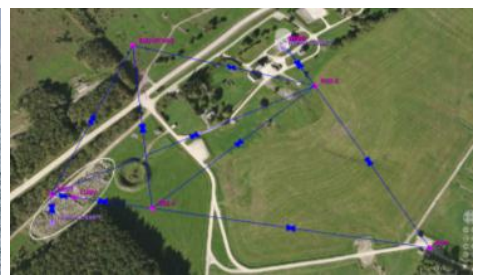

d)

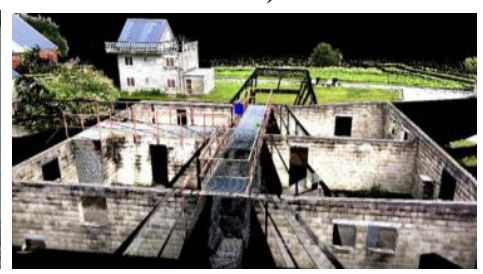

h)

Fig. 3: Operational field trials for requirements validation: a, e): Ground robots navigating indoor and outdoor on rough terrain [13]; b, f): Aerial platforms searching for human victims and doing site surveys; c, g): Maritime platforms testing deployment of rescue capsules for rescuing victims in water [10]; d): Communication tools trials [14] and h) 3D reconstruction of the environment [15] 
The land trials consisted of an exercise of an USAR intervention on a training site used by the Belgian First Aid and Support Team. The site comprises 2 areas: one area simulating a town with skeleton houses useful for indoor training and another area with a rubble field simulating a destroyed structure, with an underground tunnel system. The sea trials took place near Sesimbra, Portugal, with the main objective of testing the solutions developed so far and of obtaining feedback from end users. For that purpose, the Portuguese Navy joined together an expert panel composed by navy officers working in areas directly related to MSAR to attend part of those trials.

\section{Acknowledgements}

The research leading to these results has received funding from the European Union Seventh Framework Programme (FP7/2007-2013) under grant agreement number 285417 (ICARUS).

\section{References}

[1] G. De Cubber, D. Doroftei, D. Serrano, K. Chintamani, R. Sabino, S. Ourevitch, The EUICARUS project: developing assistive robotic tools for search and rescue operations, IEEE SSRR Symposium, 2013

[2] D. Doroftei, G. De Cubber, K. Chintamani, Towards collaborative human and robotic rescue workers, 5th International Workshop on Human-Friendly Robotics (HFR2012), October 2012

[3] S. Govindaraj, K. Chintamani, J. Gancet, P. Letier, B. Van Lierde, Y. Nevatia, G. De Cubber, D. Serrano, J. Bedkowski, C. Armbrust, J. Sanchez, A. Coelho, M.E. Palomares, I. Orbe, The ICARUS Project - Command, Control and Intelligence (C2I), IEEE SSRR, Sweden, October 2013

[4] Available on http://www.fp7-icarus.eu/sites/fp7-icarus.eu/files/publications/D100-1\%20v8.0.pdf

[5] Information on http://www.insarag.org/en/methodology/guidelines.html

[6] G. De Cubber, D. Doroftei, Y. Baudoin, D. Serrano, K. Chintamani, R. Sabino, S. Ourevitch, Operational RPAS scenarios envisaged for search \& rescue by the EU FP7 ICARUS project, Remotely Piloted Aircraft Systems for Civil Operations (RPAS2012), December 4-5, 2012

[7] Available on http://www.fp7-icarus.eu/icarus-presented-forum-do-mar-2012-presentations

[8] S. Leutenegger, M. Jabas, and R. Y. Siegwart, Solar Airplane Conceptual Design and Performance Estimation. Journal of Intelligent and Robotic Systems, Vol. 61, No. 1-4, pp. 545-561

[9] C. Armbrust, G. De Cubber, K. Berns: ICARUS -Control Systems for Search and Rescue Robots, Book on Field and Assistive Robotics, 2014, to be published.

[10] A. Matos, E. Silva, N. Cruz, J.C. Alves, D.R. Almeida, M.A. Pinto, A. Martins, J.M. Almeida, D. Cabral Machado, Development of an Unmanned Capsule for Large-Scale Maritime Search and Rescue, MTS/IEEE OCEANS 2013, San Diego, September 2013, San Diego, USA

[11] S. Omari, M.D. Hua, G. Ducard, T. Hamel, Nonlinear Control of VTOL UAVs Incorporating Flapping Dynamics, IROS, 2013

[12] Information on http://www.udt-global.com/files/marcello_zannini.pdf

[13] H. Balta, G. De Cubber, D. Doroftei, Y. Baudoin, H. Sahli, Terrain Traversability Analysis for off-road robots using Time-Of-Flight 3D Sensing, 7th IARP RISE Workshop, Russia, October 2013

[14] M. H. Chaudhary and B. Scheers. Progressive Decentralized TDMA Based MAC: Joint Optimization of Slot Allocation and Frame Lengths. In IEEE Mil. Com. Conf., USA, 2013.

[15] J. Bedkowski, G. De Cubber, A. Maslowski, 6DSLAM with GPGPU computation, in Proc. Automation 2012, Warsaw, Poland, April 2012. 